\title{
MOLTEN CARBONATE FUEL CELL MODELLING
}

\author{
Sergio Bittanti * Silvia Canevese* Antonio De Marco* \\ Gianluca Moretti* ${ }^{*}$ Valter Prandoni ${ }^{* *}$
}

\author{
* Dipartimento di Elettronica e Informazione, Politecnico di \\ Milano, Milan, Italy \\ ** CESI (Centro Elettrotecnico Sperimentale Italiano) S.p.A., \\ Milan, Italy
}

\begin{abstract}
Hybrid plants where a fuel cell and a gas turbine are combined have attracted the attention of the power system community. In this paper, a model is provided of a Molten Carbonate Fuel Cell stack and of the thermo-hydraulic equipment in which it is embedded. The model is worked out from basic physical considerations; however, it is also simple enough for simulation and control purposes. Besides, it has been validated against experimental data. Copyright ${ }^{(} 2005$ IFAC.
\end{abstract}

Keywords: Fuel Cells, Models, Power Plants, Simulators

\section{INTRODUCTION}

Power systems based on Fuel Cells (FCs) are gradually gaining interest. The main reason is the low polluting emission in terms of $\mathrm{CO}_{2}, \mathrm{NO}_{x}, \mathrm{SO}_{x}$, as well as the remarkable efficiency in energy conversion. Modularity is also a relevant issue: generators of different size can be adapted to different users' needs, and therefore to different markets. (Kowalenko, 2004), (Gurney, 2004), (Andrews and Weiner, 2004), (Evers, 2003).

Notwithstanding the importance of these systems in the present years, and in the years to come, the attention of the control community is still relatively modest. The purpose of this paper is to develop a sound mathematical model for a certain class of these systems, namely Molten Carbonate Fuel Cells (MCFCs), and to point out some of their main dynamical characteristics. This is the ground for future activity on cell control, with special reference to the project of innovative hybrid power plants (Prandoni et al., 2003), where these cells are coupled with gas turbines (also for distributed generation), in order to achieve higher total efficiency. In the absence of operating experience for such plants, in fact, a reliable model-based stack simulator can be employed to predict the be- haviour of relevant process variables (like temperature distributions, gas concentrations, overall voltage and power) and this can help to understand relationships and dependencies both among them and with variables related to the other devices connected to the FCs. This, in turn, is the starting point to study if operating constraints, tied to material limitations and requested performance, can be met, both in steady-state and in transient conditions, by means of suitable control actions, obtained, e.g., with industrial PIDs (cmp (Lukas et al., 2000)).

\section{MOLTEN CARBONATE FUEL CELLS}

FCs are stratified systems, with a usually planar or cylindrical structure (in Figure 1, a typical planar structure is represented). They work as electric generators, provided that they are continuously fed with fuel and oxidant: in fact, their two (porous) electrodes, the anode and the cathode, offer catalytic sites for the cell electrochemical reactions. Roughly speaking, hydrogen (either directly pumped into the anode or obtained by internal hydrocarbon reforming) and oxygen (at the cathode) combine to produce not only water and heat, but also a potential difference, which enables current flow in an external circuit.

An electrolyte layer, interposed between the elec- 
trodes, closes the circuit path inside the cell, allowing the transport of ions produced or consumed by the reactions occurring at the electrodes. FCs can be classified on the basis of their electrolyte; MCFCs, for instance, contain a mixture of alkali carbonates, $\mathrm{Li}_{2} \mathrm{CO}_{3}$ and $\mathrm{K}_{2} \mathrm{CO}_{3}$ mainly, which are imprisoned in an inert matrix and which, at the operating temperature, around $903-943 \mathrm{~K}$, are actually in a molten state (EG \& G Services, 2000).

Figure 1 pictures the typical structure of a MCFC. The feeding gas mixtures flow over the electrodes in perpendicular directions to each other (cross flow) and, from those bulk regions, they diffuse into the electrodes, which act as catalysts, mainly for the reactions generating or consuming electrons. The electrode layer, i.e. the porous medium, is formed in turn by sub-layers: on the one side, between electrode and gas, there is a thin porous metal plate (the porous wall), which acts as a diffusor, favouring the penetration of the gas mixture into the electrode pores; the liquid electrolyte floods the other side of the porous material, without reaching the gas side though, thanks to surface tension; inside the pores, the penetrated gas diffuses into the electrolyte and it is enabled to react with its ions: this occurs just on the pores inner surface.

The gaseous components to be considered are listed in Figure 1. At the anode, the gas-shift reaction

$$
\mathrm{CO}+\mathrm{H}_{2} \mathrm{O} \leftrightarrow \mathrm{CO}_{2}+\mathrm{H}_{2}
$$

and the electrochemical half-reaction

$$
\mathrm{H}_{2}+\mathrm{CO}_{3}^{--} \leftrightarrow \mathrm{CO}_{2}+\mathrm{H}_{2} \mathrm{O}+2 e
$$

occur, while at the cathode there is only the electrochemical half-reaction

$$
\frac{1}{2} \mathrm{O}_{2}+\mathrm{CO}_{2}+2 e \leftrightarrow \mathrm{CO}_{3}^{--} .
$$

Since each cell generates a voltage of about $1 \mathrm{~V}$ only, many elementary cells, connected electrically in series, are usually packed into a stack to obtain the required voltage, typically $100 \mathrm{~V}$. Metal plates isolate anode and cathode gases feeding adjacent elementary cells.

The system subject of this study is a prototype of a high-efficiency hybrid pilot plant, under construction at CESI; this integrates a MCFC stack, composed of $N_{C}=150$ cells and producing $125 \mathrm{kWe}$ (with a 1100 $A$ current), with a micro-size gas turbine, delivering alone about $60 \mathrm{kWe}$. An intense research activity is currently underway, including many laboratory tests, modelling efforts and studies for the investigation of plant control problems arising in normal and emergency conditions.

Here, a model of the stack and its implementation in a Matlab-Simulink@ environment are described. This

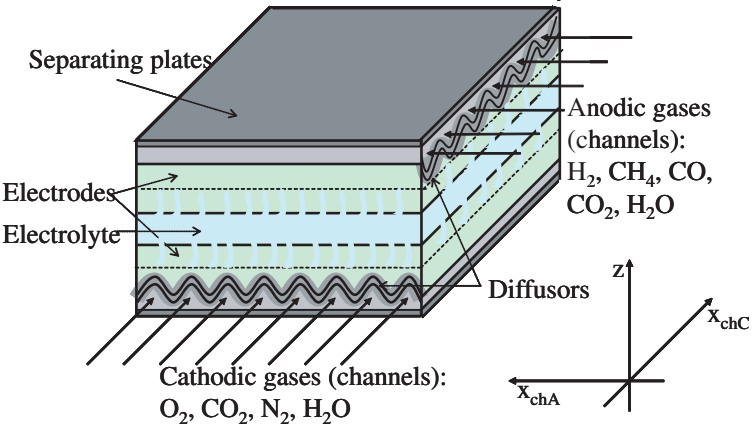

Fig. 1. The planar stratified structure of a MCFC; notice the cross-flow of anode and cathode gases

model has been connected to the models of the other devices operating with the stack, so as to form an overall dynamic simulator of the MCFC sub-plant.

The paper is organized as follows: in Section 3, a model for an elementary cell, dealing with chemical reactions, mass and energy transport and storage and electrical phenomena, is worked out; Section 4 sums up how the model has been implemented; then, model validation against steady-state real data is summarized (Section 5); finally, some transients of interest are analysed in Section 6.

\section{MODELLING OF A MCFC}

This section outlines a MCFC model which is derived from first-principle considerations, with energy, mass and momentum conservation equations plus suitable constitutive equations and electrical equations. The stack model can be obtained easily from the elementary cell model by observing that the electric current flowing through all the cells is the same, the whole stack potential difference is $N_{C}$ times that of the single cell and the total anode and cathode gas flow rates are, on the average, equally subdivided among the single cells, which they feed in parallel.

In order to avoid the typical limitations of zerodimensional and one-dimensional models, which do not take into consideration the spatial dynamical coupling between anode and cathode reaction rates due to gas cross flow, it is necessary to turn to at least a twodimensional approach (Lukas et al., 2000), (Lukas et al., 1999), (Duijn and Fehribach, 1993), (Lukas and Ghezel-Ayagh, 2001), (He, 1994): in fact, reaction rates strongly depend on local reactant concentrations and on local temperatures. We therefore divide the orthogonal gas flows into $N_{c h A}$ anodic strips, the socalled anodic channels, and into $N_{c h C}$ cathodic channels; channels are referred to orthogonal coordinates, $x_{c h A}$ and $x_{c h C}$, as shown in Figure 1. In turn, each anodic (cathodic, respectively) channel is subdivided into $N_{t A}\left(N_{t C}\right)$ volumes, or anodic (cathodic) nodes, so that in each anodic (cathodic) channel there are $N_{t A}=N_{c h C}\left(N_{t C}=N_{c h A}\right)$ control volumes. In the simulations reported later on, the assumption $N_{c h A}=4$ and $N_{c h C}=8$ is made. In the perpendicular direction 
$z$ to the anodic and cathodic flow planes, i.e. along the cell thickness, different layers have different associated control volumes, for both energy and mass conservation equations: this leads to the formulation of a final model that can be considered, in many respects, as three-dimensional (although not as far as gas velocity and pressure fields are concerned), where each control volume is identified by its "node-channellayer" coordinates.

The conservation equations describing each control volume in the various physical layers form a set of Partial Differential Equations complemented with algebraic equations.

\subsection{Thermochemical model}

Mass conservation in the bulk

Consider each single, $i-t h$ component in the anodic and cathodic gas mixtures and the $x$ coordinate along which it moves. Denoting its mass fraction by $f_{i}(x, t)$, its mass conservation equation, inside the bulk, for an elementary control volume with area $A$, is

$$
\rho A \frac{\partial f_{i}}{\partial t}+w \frac{\partial f_{i}}{\partial x}=w_{l t}^{\prime}\left(f_{i}^{*}-f_{i}\right)+H_{g d}\left(f_{i}^{*}-f_{i}\right)
$$

where, in the first member, there are the usual accumulation and convective transport terms and, in the second one, the contribution given by lateral flows between the channel and the porous medium. $w_{l t}^{\prime}$ is the total lateral convective gas flow rate, per unit length, at the diffusor-channel interface. On the anode side, the lateral total flow rate should, in ordinary operating conditions, go from the porous medium towards the bulk; therefore, $f_{i}^{*}=f_{i p w}$ ( $p w$ stands for porous wall). On the cathode side, instead, the lateral total flow rate should always (under correct operation) be directed from the bulk towards the porous medium; thus, $f_{i}^{*}=f_{i}$.

Mass conservation in the catalyst

For the $i-t h$ gaseous component inside the porous medium (subscript $\mathrm{pm}$ in the following), in the anodic and cathodic layers which are not completely filled by the electrolyte ( $\varepsilon_{g}$ will be the space filling coefficient, in the porous medium, by the gas mixture), there are the accumulation, diffusion and convection terms along $z$ and the terms of (electro)chemical production/consumption of the component:

$$
\begin{gathered}
\varepsilon_{g} A_{p} \frac{\partial}{\partial t}\left(\rho f_{i_{p m}}\right)+\varepsilon_{g} \frac{\partial}{\partial z}\left(w_{p} f_{i_{p m}}\right)=\gamma_{d i f f_{i}} \varepsilon_{g} A_{p} . \\
\cdot \frac{\partial}{\partial z}\left(\frac{\partial \rho f_{i_{p m}}}{\partial z}\right)+\left(\dot{R}_{i, s h} A_{s h}+\frac{i_{i}^{\prime}}{2 F}\right) P M_{i}
\end{gathered}
$$

where $P M_{i}$ is the component molar mass. The shift reaction conversion rate $\dot{R}_{i, s h}\left[\mathrm{kmol} / \mathrm{m}^{3} \mathrm{~s}\right]$ can be calculated by multiplying by density the classical rate (Xu and Froment, 1989) function of partial pressures,
Gibbs energy and temperature. Electrochemical dynamics, not considered here for simplicity, as observed in Section 3.3, are object of current work, based on the analysis of ionic motion in the electrolyte and, near the electrode pore surfaces, of the formation of potential differences due to the presence of charged activated complexes. This other approach, in fact, aims at describing the origin of capacitive effects, which are responsible for cell fast dynamics, developing on a time scale around 1 second or less.

\section{Boundary conditions for mass}

For each electrode, at the bulk-diffusor interface, a sort of radiation condition can be written:

$$
H_{g d}\left(f_{i}-f_{i_{p m}}(0, t)\right)+\left.D_{d} \Omega_{g d} \frac{\partial\left(\rho f_{i_{p m}}\right)}{\partial z}\right|_{z=0}=0
$$

where mass convective motion and gas mass diffusion towards the catalyst inner layers appear. $D_{d}$ is a suitable mass diffusion coefficient in the porous medium. The boundary conditions of the layers completely filled by the electrolyte are null mass fluxes.

\section{Energy conservation in the bulk}

We consider, for each channel, thermal energy accumulation, energy convective transport along $x$, work of pressure and friction forces, energy flux between channel and porous medium (tied to temperature differences between the gas bulk in the channel and the diffusor), energy flux (analogous to the previous one) between channel and separating plate, energy flux due to convective flow rates between each channel and the two adjacent ones. The energy flux due to heat diffusion along $x$ and to matter diffusion from channel to porous medium is neglected. Considering mass and momentum conservation as well, after some manipulations the energy equation becomes

$$
\begin{aligned}
& \rho A \frac{\partial e}{\partial t}+w \frac{\partial h}{\partial x}=\Gamma_{g d}\left(T_{d}-T\right)+\Gamma_{g p}\left(T_{p}-T\right)+ \\
& +w_{l t}^{\prime}\left(e^{*}-e\right),
\end{aligned}
$$

where $T(x, t), e(x, t), h(x, t)$ are, for the bulk gas in the channel, temperature, internal specific energy and specific enthalpy respectively; $T_{d}$ and $T_{p}$ are the diffusor temperature at the channel interface and the plate temperature; $\Gamma_{g d}$ and $\Gamma_{g p}$ are the heat exchange coefficients per unit length $(W /(m K))$ between gas in the channel and, respectively, the diffusor and the plate; $e^{*}=e\left(T_{d}\right)$ for anodic channels, $e^{*}=e$ for cathodic channels.

Energy conservation in the catalyst and in the electrolyte

The porous medium on the gas side can be roughly modelled as a combination of two elements: the electrode material itself (a nickel alloy for the anode and lithiated nickel oxide, a highly conductive semiconductor, for the cathode) and the gas diffusing into the pores. For those layers where the gas penetrates and where reactions can take place, we consider ther- 
mal energy accumulation, energy convective transport by gas, thermal energy diffusive transport, (electro)chemical generation, generation by Joule's effect (due to the passing of electric current) and lateral (with respect to $z$ ) diffusive energy fluxes. The equations about the porous medium layers which are adjacent to the electrolyte (where the electrolyte leaks) and the ones relative to the electrolytic layer, which separates the electrodes, are analogous to these ones, but without the reaction terms (for the complete equation list, refer to (Moretti, 2003)).

\section{Boundary conditions for energy}

For each electrode, at the channel-diffusor interface, this radiation condition stands:

$$
\Gamma_{g d} A_{p}\left(T-T_{p m}(0, t)\right)=-\left.\lambda_{d} \Omega_{g d} \frac{\partial T_{p m}}{\partial z}\right|_{z=0},
$$

where $\lambda_{d}$ is the diffusor thermal conductivity.

Diffuser and electrode constitute a unique, but inhomogeneous, porous medium: therefore, temperature gradients are continuous in the passage from the one to the other, so that no boundary condition is needed between them: only physical parameters vary.

\subsection{Hydrodynamics}

For each anodic and cathodic channel, we take global mass conservation, i.e.

$$
\frac{\partial(\rho A)}{\partial t}+\frac{\partial w}{\partial x}=w_{l t}^{\prime}
$$

and general momentum conservation:

$$
\frac{\partial w}{\partial t}+\frac{\partial w u}{\partial x}+A \frac{\partial p}{\partial x}=\tau_{f r d} \Omega_{g d}+\tau_{f r p} \Omega_{g p} .
$$

In the latter, on the left, there are the two inertial terms and the term due to pressure forces; on the right, the terms due to tangential friction on diffusor and plate (the gravity term is negligible). These equations, yielding all flow rates and pressures along the channels as functions of the anode and cathode inlet and outlet pressures, are integrated over finite control volumes which are staggered with respect to the ones considered for the single component mass equations and for energy equations.

In the porous medium layers, pressures are supposed to be equal to the pressure in the corresponding bulk control volume, while flow rates can be computed through the total mass conservation equation: at each porous wall, the flow rate must vanish, while across the surface between the diffuser and the active layer the flow rate is linked, of course, to electric current.

At every instant and in every control volume, the lateral flow rates, to or from the porous medium, supply a known contribution $\left(w_{l t}^{\prime}\right)$ to the mass conservation equation.

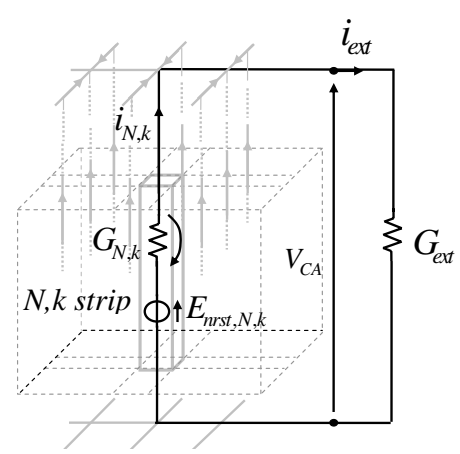

Fig. 2. The structure assumed for the electrical model

\subsection{Electrical equations}

From the electrical point of view, a single cell acts as the parallel of elementary real generators, each of which is a fraction of the cell thickness from the anode to the cathode: this is shown schematically in Figure 2, where the generic $(N, k)$ strip $\left(N=1,2, \ldots, N_{c h A}, k=\right.$ $\left.1,2, \ldots, N_{c h C}\right)$ is highlighted and the external circuit is assumed, for simplicity, to be a plain conductance $G_{\text {ext }}$. The total current, flowing through the cell and the external circuit, and the potential difference are, respectively,

$$
\begin{gathered}
i_{\text {ext }}=\sum_{N=1, k=1}^{N_{c h A}, N_{c h C}} i_{N, k} \\
V_{C A}=E_{n r s t, N, k}-\frac{i_{N, k}}{G_{N, k}}=\frac{i_{e x t}}{G_{e x t}}
\end{gathered}
$$

whence

$$
V_{C A}=\frac{\sum_{N=1, k=1}^{N_{c h A}, N_{c h C}} E_{n r s t, N, k} G_{N, k}}{G_{e x t}+\sum_{N=1, k=1}^{N_{c h A}, N_{c h} C} G_{N, k}}
$$

where $E_{n r s t, N, k}$ is the Nernst potential associated to the $(N, k)$ strip.

In general, $V_{C A}$ is a function both of $i_{e x t}$ and of external control variables which depend on the nature of the external circuit (think, e.g., of a stack connected to a network through a DC-DC converter connected to an inverter).

The Nernst potential is the open circuit reversible voltage between the electrodes and it is a function of local temperature and of reactant molar fractions in the reaction zones just inside the liquid electrolyte: in every strip, omitting subscripts $N$ and $k$ for simplicity,

$$
E_{n r s t}-E^{0}=\frac{R T}{2 F} \ln \frac{\left[\mathrm{O}_{2}\right]_{c}^{\frac{1}{2}}\left[\mathrm{CO}_{2}\right]_{c}\left[\mathrm{H}_{2}\right]_{a}\left[\mathrm{CO}_{3}^{--}\right]_{a}}{\left[\mathrm{CO}_{3}^{--}\right]_{c}\left[\mathrm{H}_{2} \mathrm{O}\right]_{a}\left[\mathrm{CO}_{2}\right]_{a}},
$$

where $E^{0}$ is the standard conditions potential and where the neutral component concentrations refer to the gas diffused into the liquid electrolyte. Anyway, neglecting the limits due to neutral components diffusion into the electrolyte and the transport of $\mathrm{CO}_{3}^{--}$ 
from the anode to the cathode (by assuming that $\left.\frac{\left[\mathrm{CO}_{3}^{--}\right]_{a}}{\left[\mathrm{CO}_{3}^{--}\right]_{c}}=1\right)$, one has

$$
\begin{aligned}
E_{n r s t}-E^{0}= & \frac{R T_{a}}{2 F} \ln \frac{\left[H_{2}\right]_{a}}{\left[H_{2} O\right]_{a}\left[C O_{2}\right]_{a}}+ \\
& +\frac{R T_{c}}{2 F} \ln \left(\left[O_{2}\right]_{c}^{\frac{1}{2}}\left[C O_{2}\right]_{c}\right),
\end{aligned}
$$

where the (anodic and cathodic) gas concentrations are employed, which simplifies model implementation. This approximation, which is fair in stationary conditions, is anyway usually extended to quasistationary conditions too (EG \& G Services, 2000). It stands in the present work as well, since the main control objectives that one can figure out for the whole MCFC-microturbine plant involve relatively slow dynamics (e.g., among operating constraints there is keeping cell and combustor temperatures under certain values), so that (electro-)chemical phenomena can be considered as having already reached their steady state and therefore neglected in the description: the high-frequency effects due to charge storage inside the FCs, in fact, become relevant only when control of the external electrical system, which exhibits very small time constants alike, is included.

\section{IMPLEMENTATION OF THE GLOBAL MODEL}

The equations described so far draw an overall picture of a single MCFC. Inside every $(N, k)$ control volume in the cell, the bulk equations are integrated along the anodic and cathodic $x$ coordinates, those regarding the catalytic medium are integrated along coordinate $z$ (cmp (Leva and Maffezzoni, 2003), (Eitelberg, 1983)): therefore, by using average variables in each volume, ODEs are obtained from PDEs. These ODEs are then time-discretized (with sampling intervals of $0,1 \div 1$ second), yielding approximated equations which are finally solved mostly with an iterative implicit method, since there are nonlinearities and in order to cope with strong ties among some equations and with equation subsets where slow and fast dynamics interact. As an example of the numerical method adopted, let us consider equation (1): first, it can be transformed into

$$
\begin{aligned}
& \rho_{N, k} A \Delta x_{N, k} \frac{\partial f_{i, N, k}}{\partial t}+\max \left(0, w_{N, k^{\prime}}\right)\left(f_{i, N, k}-f_{i, N, k^{\prime}}\right)= \\
& =w_{l t, N, k}^{\prime}\left(f_{i, N, k}^{*}-f_{i, N, k}\right)+H_{g d} \Delta x_{N, k}\left(f_{i, N, k}^{*}-f_{i, N, k}\right),
\end{aligned}
$$

where subscripts $N, k$ denote the average quantities in volume $(N, k)$, subscripts $N, k^{\prime}$ denote quantities at the edge between volume $(N, k)$ and volume $(N, k-1)$ and $\Delta x_{N, k}$ is the length of the integration interval along the integration direction $x$ ( $k$ is taken as the discrete spatial coordinate along $x$ ). Then, the implicit Euler method is used to time-discretize the approximate equation, and the final unknowns are $f_{i, N, k}^{*}, f_{i, N, k^{\prime}}$ and $f_{i, N, k}$ at the new time instant $t_{n+1}$, while the same variables are assumed to be known at the old instant $t_{n}$. This equation is linear in the unknowns, so it can be solved directly; for nonlinear equations, it is enough to apply an iterative method, by linearizing, at each step, around the unknowns.

The numerical algorithm just described has been implemented in the $\mathrm{C}++$ language and embedded in a

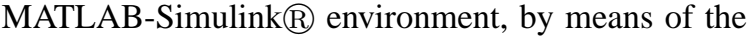
S-Function functionality.

The cell simulator can be employed to study the dynamics of the main physical phenomena. Moreover, it can help to plan suitable operating manoeuvres and control strategies for the cell. However, these last cannot, in many cases, be conceived without an analysis of cell behaviour within its plant, whence the need to model also the other components of the MCFC system considered here.

Therefore, a thermodynamic model for each kind of device in the process, based, again, on conservation principles and constitutive equations, has been built. In particular, each one-dimensional element is first divided into control volumes (nodes), along its curvilinear abscissa; then, single component mass and energy conservation equations (thermochemical equations) for the fluid in each node are integrated and the thermodynamical properties of the fluid are substituted with the thus obtained average values; the momentum and global mass conservation equations (hydrodynamic equations), instead, are integrated on branches, i.e. control volumes staggered with respect to the nodes.

System topology, i.e. the corresponding net of element connections, is described by an incidence matrix, supplying, for each ordinary node, corresponding to a physical device, the list of entering and outgoing branches, and, for each ordinary branch, the upstream and downstream nodes; the net boundary conditions (pressure, temperature, composition) are described by fictitious terminal nodes and branches.

In the overall integration technique, external nodes, whose equations and unknown hydrodynamic variables actually participate in building up the global hydrodynamic system, are distinguished from inner nodes, whose equations are solved locally in the single macro-branch (Dell'Oca, 1999). This way, local hydrodynamics (local with respect to the single control volumes) is handled so as to maintain stability and, at same time, reduce the total hydrodynamic system dimension, thus leading to shorter simulation times; in other words, the problem of slow dynamics interacting with fast dynamics, which arises when the nodes in a net are numerous and many of them correspond to quite small control volumes, making the total hydrodynamic system stiff, with consequent lack of convergence and/or stability, is avoided.

The resolution of the equations describing the elementary devices has been carried out by decoupling thermal equations, chemical equations and, for branches 


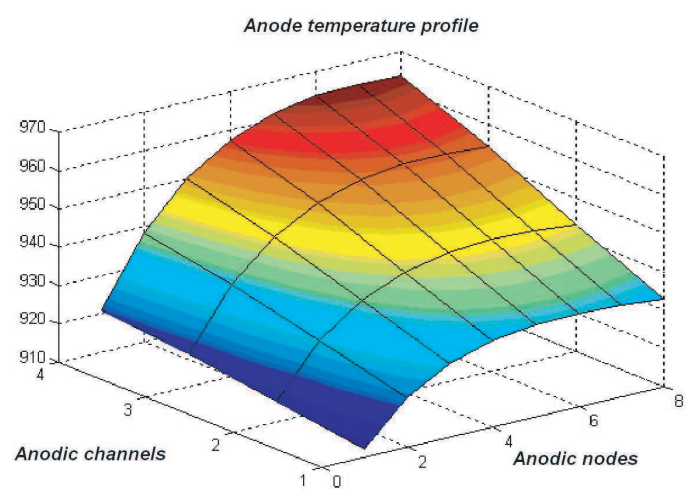

Fig. 3. Anode temperature profile

and macro-branches, hydrodynamics from the hydrodynamic equations for the nodes. This decoupling concurs to reduce calculation times and it is possible because transport phenomena of thermal and chemical quantities occur with the fluid speed, while transport phenomena of hydrodynamic quantities develop at sound speed (Colombo et al., 1989).

\section{MODEL VALIDATION}

The cell model has been validated against experimental results (Scagliotti et al., 1999), about a MCFC stack composed of 14 active cells with $700 \mathrm{~cm}^{2}$ useful area each. We refer to three stationary conditions:

(1) open circuit;

(2) $70 \mathrm{~A}$ current, $642 \mathrm{~W}$ power;

(3) $70 \mathrm{~A}$ current, $700 \mathrm{~W}$ power.

A test at working point 2 was exploited empirically to identify some of the model uncertain parameters, like exchange coefficients and the internal electrical resistance, so that the implemented cell model could lead to numerical results coherent with experimental data. The thus obtained parameter values were then held fixed in the other tests.

In all the three cases, the imposed inputs were the pressures and the temperatures of the inlet anodic and cathodic gases and the related mass fractions.

As for case 1, the obtained values are shown in Table 1. Figures 3 and 4 show the temperature profile of

Table 1. First case

\begin{tabular}{lll}
\hline & $\begin{array}{l}\text { Experimental } \\
\text { data }\end{array}$ & $\begin{array}{l}\text { Calculated } \\
\text { data }\end{array}$ \\
\hline Average cell voltage & $(927,4 \pm 0,9) \mathrm{mV}$ & $926,8 \mathrm{mV}$ \\
Anode outlet temperature & $\sim 925 \mathrm{~K}$ & $928 \mathrm{~K}$ \\
Cathode outlet temperature & $\sim 925 \mathrm{~K}$ & $928 \mathrm{~K}$ \\
\hline
\end{tabular}

the bulk plates at the anode and at the cathode of an elementary cell. In cases 2 and 3, respectively, the obtained results are summarized in Tables 2 and 3 .

Analogous tests, carried out with other experimental data (Previtali and Villa, 2002), have allowed a validation of the parts of the model relative to the reformer

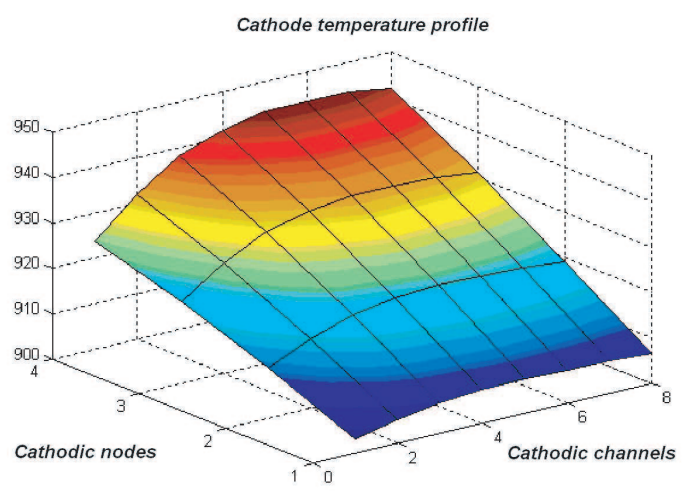

Fig. 4. Cathode temperature profile

Table 2. Second case

\begin{tabular}{lll}
\hline & $\begin{array}{l}\text { Experimental } \\
\text { data }\end{array}$ & $\begin{array}{l}\text { Calculated } \\
\text { data }\end{array}$ \\
\hline $\mathrm{H}_{2}$ consumption (anode) & $60 \%$ & $60,4 \%$ \\
$\mathrm{CO}_{2}$ consumption (cathode) & $22 \%$ & $21,7 \%$ \\
Anode outlet temperature & $\sim 945 \mathrm{~K}$ & $948 \mathrm{~K}$ \\
Cathode outlet temperature & $\sim 945 \mathrm{~K}$ & $938 \mathrm{~K}$ \\
\hline
\end{tabular}

Table 3. Third case

\begin{tabular}{lll}
\hline & $\begin{array}{l}\text { Experimental } \\
\text { data }\end{array}$ & $\begin{array}{l}\text { Calculated } \\
\text { data }\end{array}$ \\
\hline $\mathrm{H}_{2}$ consumption (anode) & $51 \%$ & $51,22 \%$ \\
$\mathrm{CO}_{2}$ consumption (cathode) & $\sim 22 \%$ & $21,5 \%$ \\
Anode outlet temperature & $\sim 945 \mathrm{~K}$ & $946 \mathrm{~K}$ \\
Cathode outlet temperature & $\sim 945 \mathrm{~K}$ & $937 \mathrm{~K}$ \\
\hline
\end{tabular}

and the combustor supporting the MCFC stack. For conciseness, anyway, those results are not reported here.

\section{TRANSIENT SIMULATIONS}

After validation, the implemented model was employed to simulate the transient behaviour of the 150 cell stack (useful area of each cell: $0,8 \mathrm{~m}^{2}$ ) under construction (Prandoni et al., 2003). In particular, starting from a stationary situation of open circuit at the normal exercising temperature, the stack was supposed to get instantaneously connected with an external load (at time $t=50$ seconds), a simple conductance, in order to drive the produced nominal power to approximately $120 \mathrm{~kW}$. The imposed inputs are reported in Table 4.

Table 4. Simulation inputs

\begin{tabular}{lll}
\hline & Anode & Cathode \\
\hline Inlet pressure & $3,498 \mathrm{E} 5 \mathrm{~Pa}$ & $3,516 \mathrm{E} 5 \mathrm{~Pa}$ \\
Outlet pressure & $3,479 \mathrm{E} 5 \mathrm{~Pa}$ & $3,493 \mathrm{E} 5 \mathrm{~Pa}$ \\
Inlet gas temperature & $870 \mathrm{~K}$ & $875,89 \mathrm{~K}$ \\
Inlet $\mathrm{CO}_{2}$ mass fraction & 0,20931 & 0,02901 \\
Inlet $\mathrm{H}_{2}$ mass fraction & 0,07031 & 0,0 \\
Inlet $\mathrm{N}_{2}$ mass fraction & 0,0 & 0,72337 \\
Inlet $\mathrm{CO}$ mass fraction & 0,14806 & 0,0 \\
Inlet $\mathrm{CH}_{4}$ mass fraction & 0,0389 & 0,0 \\
Inlet $\mathrm{H}_{2} \mathrm{O}$ mass fraction & 0,53341 & 0,07021 \\
Inlet $\mathrm{O}_{2}$ mass fraction & 0,0 & 0,17741 \\
\hline
\end{tabular}




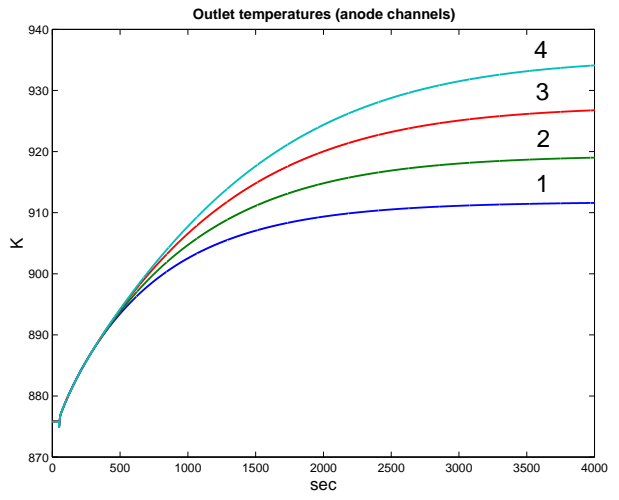

Fig. 5. Outlet temperature of the anode channels versus time

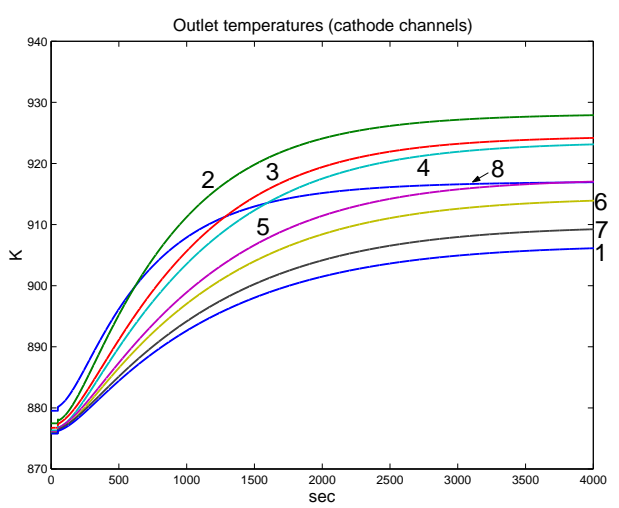

Fig. 6. Outlet temperature of the cathode channels versus time

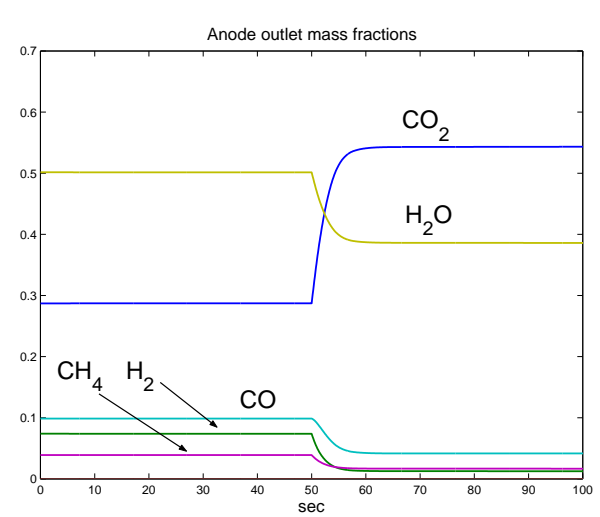

Fig. 7. Anode outlet mass fractions versus time

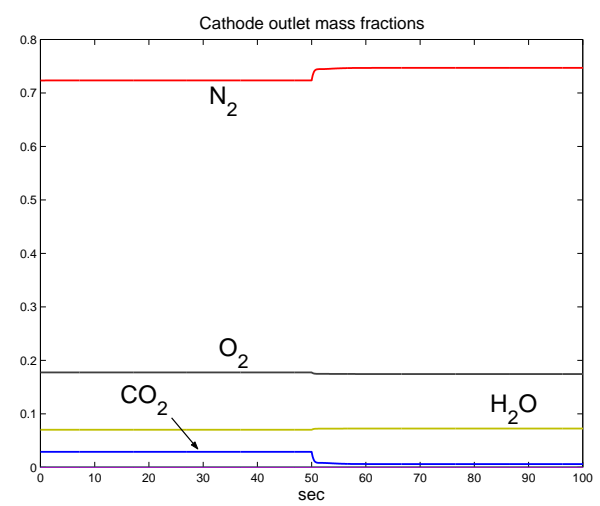

Fig. 8. Cathode outlet mass fractions versus time

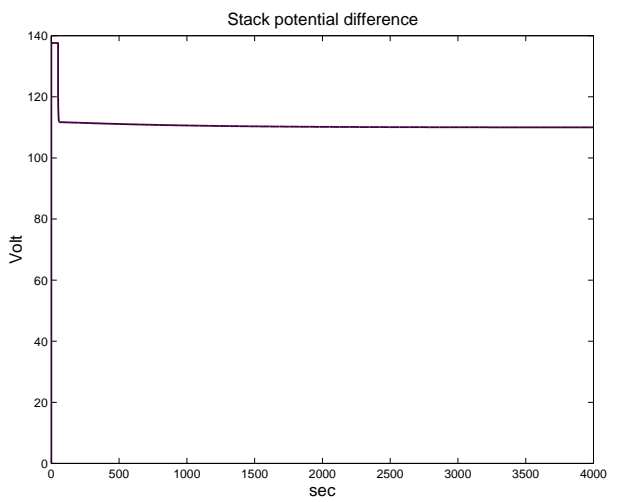

Fig. 9. Stack voltage versus time

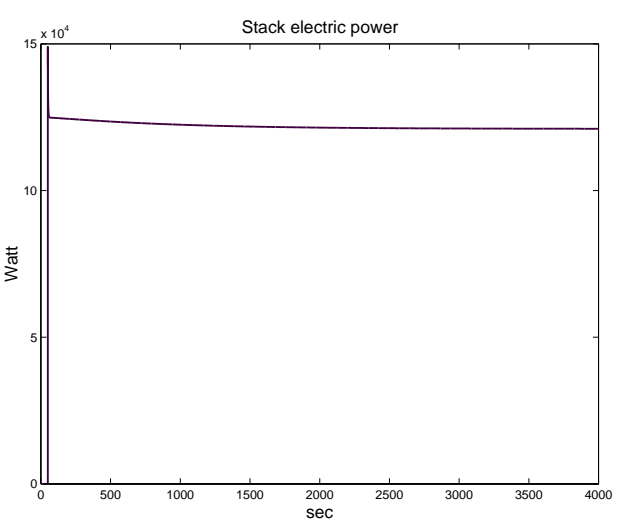

Fig. 10. Stack electrical power versus time

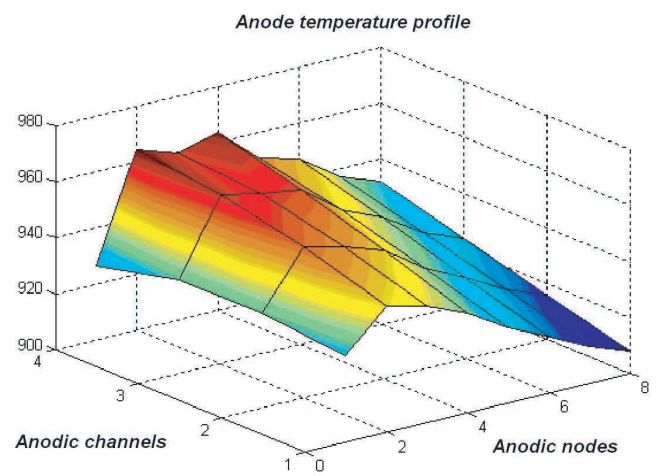

Fig. 11. Anode temperature profile

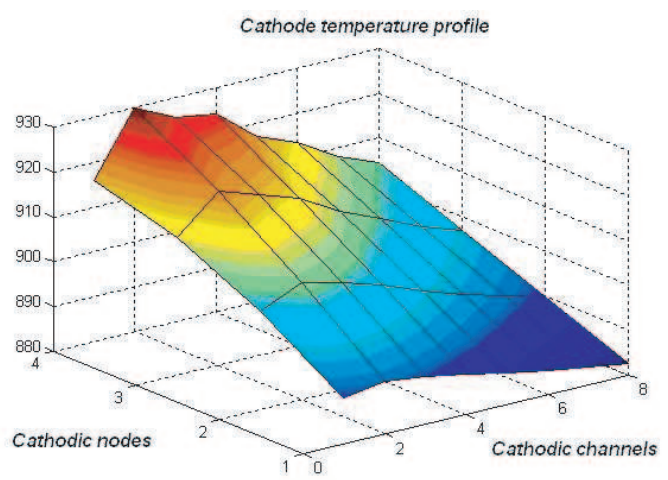

Fig. 12. Cathode temperature profile 
The obtained results are shown in Figures 5, 6, 7, $8,9,10,11,12$, where the numbers on the curves correspond to the channels (from 1 to 4 for the anode, from 1 to 8 for the cathode). In particular, the outlet temperature increase with time shows that the total electrochemical reaction is exothermal; moreover, the anodic $\mathrm{CO}_{2}$ mass fraction increase, the $\mathrm{H}_{2}$ decrease and the cathodic $\mathrm{CO}_{2}$ decrease correspond to the fact that the electrochemical reactions must shift to the right when the generators produce electrical power.

Different orders of magnitude in raising time (i.e. the time which is necessary for a variable to attain a new regime) appear clearly: time scales are of the order of thousands of seconds ( 2 or 3 hours) for temperatures, the slowest quantities, while only of tens of seconds for mass fractions, seconds for the total voltage and power.

\section{CONCLUSIONS AND FUTURE DEVELOPMENTS}

This article introduces the main aspects of a model developed for a MCFC (stack). The model describes the main physical phenomena involved and allows the computation of the electric current, power, chemical composition, temperatures at various points, etc.; besides, it is accurate enough (since it is two-threedimensional) to verify the constraints on process variables which the control system has to satisfy for plant operational procedures, in both normal and emergency conditions. Of course, since the control system refers to the whole plant, this cell model, as already mentioned, has to be integrated with the models of the other plant components (e.g. combustor and reformer). In order to increase the cell model accuracy, attention is currently focused on reaction kinetics, so that the chemical relationship too (and not only the thermal and electrical ones) between gas mixtures and electrolyte can be pointed out. Such study also includes the carbonate ion motion across the electrolyte, with its consequences on the electrical part of the model (on the Nernst equation in particular) and, in general, on system dynamics, which is so important for control purposes, for instance when the cell stack has to face fast and large changes in its electrical load.

\section{ACKNOWLEDGEMENTS}

This work has been developed in the frame of the research on the Italian Electrical System "Ricerca di Sistema", Ministerial Decrees of January 26, 2000 and April 17, 2001.

Research has been supported also by the Italian National Research Project "New Techniques of Identification and Adaptive Control for Industrial Systems" and partially by CNR-IEIIT.

\section{REFERENCES}

Andrews, C.J. and S.A. Weiner (2004). Visions of a hydrogen future. IEEE Power \& Energy Magazine 2(2), 26-34.

Colombo, P., A. De Marco, D. Laudato and V. Prandoni (1989). Modeling methods for ENEL's 320 fossil fired power plant advanced training simulator. 7th Power plant dynamics, Control and Testing Symposium, Knoxville, USA.

Dell'Oca, M. (1999). Ambiente di simulazione e modelli matematici di processi termoidraulici. Master Thesis, Politecnico di Milano, Milan, Italy.

EG \& G Services, Parsons Inc. (2000). Fuel Cell Handbook (5th Edition). U.S. Dept of Energy, Office of Fossil Energy, National Energy Technology Laboratory.

Eitelberg, E. (1983). A simple A2-Stable Numerical Method for State Space Models with Stiff Oscillations. Math. Comput. Simulation 25(4), 346-355.

Evers, A.A. (2003). Go to where the market is! Challenges and opportunities to bring fuel cells to the international market. Int. J. Hydrogen Energy 28(7), 725-733.

Gurney, J.H. (2004). Building a case for the hydrogen economy. IEEE Power \& Energy Magazine 2(2), 35-39.

$\mathrm{He}$, W. (1994). The dynamic performance of a molten carbonate fuel cell in power-generation system. J. Power Sources 52(2), 179-184.

Kowalenko, K. (2004). Getting a handle on hydrogen. IEEE - The Institute 28(3).

Leva, A. and C. Maffezzoni (2003). Modelling of power plants. In: Thermal Power Plant Simulation and Control (D. Flynn, Ed.). Vol. 2. pp. 17-60. IEE Power \& Energy Series 43.

Lukas, M. and H. Ghezel-Ayagh (2001). An explicit dynamic model for direct reforming carbonate fuel cell stack. IEEE Trans. Energy Conv. 16(3), 289-295.

Lukas, M., H. Ghezel-Ayagh and K.Y. Lee (1999). Development of a stack simulation model for control study on direct reforming molten carbonate fuel cell power plant. IEEE Trans. Energy Conv. 14(4), 1651-1657.

Lukas, M., H. Ghezel-Ayagh and K.Y. Lee (2000). Modeling, simulation and control of direct reforming molten carbonate fuel cell power plant. IFAC Symp. Power Plants and Power Systems Control 2000, Bruxelles, Belgium, 127-132.

Moretti, G. (2003). Molten Carbonate Fuel Cell: Modellistica e simulazione. Master Thesis, Politecnico di Milano, Milan, Italy.

Prandoni, V., S. Spelta, O. Lamquet, R. Massa and F. Parodi (2003). Dynamic model of a hybrid plant based on MCFC fuel cells and microturbine for process analysis and control. IEEE PowerTech 2003, Bologna.

Previtali, G. and M. Villa (2002). Modellistica e simulazione di reattori catalitici per reazioni fra componenti in fase gassosa. Master Thesis, Politecnico di Milano, Milan, Italy.

Scagliotti, M., G. Strobino, P. Araldi and P. Savoldelli (1999). Sperimentazione di una soluzione innovativa per la configurazione di stack MCFC. Giornata di studio sulle Pile a Combustibile, AIM, Milan, Italy.

Van Duijn, C.J. and J.D. Fehribach (1993). Analysis for a molten carbonate fuel cell. Electronic J. Differential Equations $1993(6), 1-25$.

Xu, J. and G.F. Froment (1989). Methane steam reforming, methanation and water-gas shift: Intrinsic kinetics. AIChE J. 35(1). 